\title{
Index to Volume 16, 2014
}

$\begin{array}{lr}\text { A } & \\ \text { acceptance } & 330-36 \\ \text { acute epiglottitis } & 184-85 \\ \text { admission } & 353-58 \\ \text { adolescent } & 123-25 \\ \text { agenda } & 73-82 \\ \text { agreement } & 227-32 \\ \text { airway } & 317-20 \\ \text { aminophylline } & 392-96 \\ \text { analgesia } & 279-88 \\ \text { anion gap } & 249-52 \\ \text { aortic dissection } & 59-62 \\ \text { aortic valve replacement } & 59-62 \\ \text { arterial puncture } & 18-23 \\ \text { asthma } & 8-17 \\ \text { asthma therapy } & 101-8 \\ \text { asystole } & 392-96 \\ \text { automatic external defibrillation }\end{array}$

270-78

avulsion fracture

\begin{tabular}{lr} 
competencies & $24-33$ \\
complications & $18-23$ \\
computed tomography & $59-62$, \\
$113-15,161-66,167-74$, & $184-85$, \\
& $261-69$ \\
consensus & $73-82$ \\
consultants & $42-51$ \\
continuing medical education \\
\multicolumn{2}{c}{$34-41$} \\
croup & $8-17$ \\
& \\
D & \\
D-dimer & \\
decision support techniques & $261-69$ \\
delayed tamponade & $186-89$ \\
Delphi & $24-33$ \\
diagnostic errors & $261-69$ \\
diagnostic imaging & $255-60$ \\
dipyridamole & $392-96$
\end{tabular}

B

back pain

bronchiolitis

bronchogenic cyst

burnout

bystander resuscitation

C

Canada

$181-83$

Canadian Triage and Acuity Scale

(CTAS)

227-32, 353-58

cardiac arrest

cardiac surgery

cerebral gas emboli

children

$270-78$

$59-62$

$385-88$

1-5, 289-99

chronic pain

52

classroom-based education $270-78$

clinical competence

241-48

clinical decision making $\quad 314-15$

clinical decision rule 300-302

clinical intervention 101-8

clinical skills

253-54

389-91

$385-88$

$42-51$

commercial aircraft

communication

community-associated methicillinresistant Staphylococcus aureus

\section{E}

educational opportunities 181-83

effectiveness 109-12

elbow dislocation 389-91

emergency 317-20

emergency department 134-40, 289-99, 303-6

emergency department

overcrowding

$198-205$

emergency medical services 73-82, $83-89,214-26$

emergency medicine 1-5, 24-33, 34-41, 42-51, 161-66, 214-26, 233-36, 241-48, 261-69

emergency physicians 321-29

emergency service 214-26

emphysematous abscess 184-85

end of life

190

extubation

303-6

F

flank pain 167-74

focused emergency department ultrasonography 186-89

frequent emergency department users education 24-33, 270-78

emergency care $\quad 8-17$
G

glue sniffing 249-52

Gray-Allen E1-E2

guidelines $\quad 1-5,314-15$

guidewire 18-23

H

health care costs 214-26

health care quality, access, and

evaluation 214-26

health policy 34-41

health service research 101-8

health services 206-13

health services' needs and

demands 214-26

hémothorax retardé $\quad 337-44$

hémothorax tardif 337-44

hip pain 123-25

hospital 214-26

hospital emergency service 345-52

humanism 175-77

humanities E1-E2, 190

humour E1-E2

\section{I}

iatrogenic 389-91

impalement thigh injury $\quad 237-38$

infectious diseases $\quad 90-100$

inferior epigastric artery rupture

influenza

$119-22$

instructional method $\quad 178-80$

interphysician communication

42-51

interrater reliability $\quad 353-58$

intravenous injection $\quad 345-52$

intubation $\quad 317-20$

L

learning 109-12

left without being seen 289-99

lesser trochanter 123-25

long-term care 206-13

low cerebrospinal fluid pressure headache

$53-58$

lumbar puncture 113-15

M

manpower 134-40

marginalized populations $90-100$,

279-88

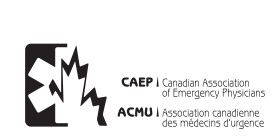


medical education 178-80, 241-48 medically underserved area 34-41 Memorial Award

190 metabolic acidosis 249-52

methicillin-resistant Stapbylococcus aureus

MIBI

mixed methods

multimedia

\section{$\mathbf{N}$}

\section{nephrolithiasis}

neuroradiology

noir

nurse practitioner

\section{O}

ophthalmology

$311-13$

osmolal gap

249-52

overuse of health services

\section{$\mathbf{P}$}

pain management $\quad 90-100$

paramedics

$206-13$

patient safety

279-88, 303-6

pediatric emergency department

$101-8$

pediatric emergency medicine

$330-36$

photography

$345-52$

physician assistants

physician attitudes

$134-40$,

321-29, 330-36

321-29

physician practice patterns 34-41

physician storage area 34-41

pneumothorax retardé 337-44

pneumothorax tardif 337-44

postural headache

PowerPoint procedural learning

procedural sedation

253-54

pulmonary embolism

279-88

300-302

\section{Q}

qualitative research

83-89

quality

$1-5,8-17,255-60$

\section{$\mathbf{R}$}

radial neck fracture

radiography

radiology

389-91

$8-17$

randomized controlled trial 345-52

rectus sheath hematoma 119-22

referral and consultation $\quad 42-51$

reflection

175-77

reliability

renal colic

227-32

$167-74$

research

$73-82$

research agenda

83-89

research education

233-36

233-36

$175-77$

residency training

resident

233-36

retinal disorders

311-13

revised Geneva score

rules

300-302

$314-15$

rural health services

$34-41$

S

salbutamol inhalation 101-8

self-directed education $\quad 270-78$

simplified revised Geneva score

$300-302$

simplified Wells rule

$300-302$

simulation

253-54

skin infection

$141-60$

soft tissue infection 141-60, 345-52 spontaneous intracranial

hypotension $\quad 53-58$

stab wound $\quad 186-89$

staffing $\quad 134-40$

Staphylococcus aureus 141-60

subarachnoid hemorrhage 113-15

superior vena cava syndrome 59-62

syndromic surveillance 198-205

$\mathbf{T}$

tar burn 307-10

tar removal 307-10

teaching $\quad 178-80$

technology 178-80

thermal injury 52

toluene 249-52

traumatisme thoracique $\quad 337-44$

triage

289-99

U

ultrasonography $\quad 18-23,119-22$

undergraduate 24-33

utilization 161-66, 255-60

utilization rate 161-66

V

vascular access $\quad 18-23$

visual loss 311-13

vitamin $\mathrm{E}$ ointment $\quad 307-10$

W

Wells rule 300-302

wilderness medicine 181-83

wind down E1-E2

$\mathbf{X}$

x-ray

261-69 